\title{
A study to determine influential factors on implementation of management information system
}

\author{
Naser Azad*, Tahereh Zamani and Seyed Foad Zarifi
}

Department of Management, Islamic Azad University, South Tehran Branch, Tehran, Iran

\begin{tabular}{l}
\hline C H R O N I C L E \\
\hline Article history: \\
Received May 1, 2013 \\
Received in revised format \\
10 July 2013 \\
Accepted 12 July 2013 \\
Available online \\
August 12013 \\
\hline Keywords: \\
MIS \\
Factor analysis \\
Banking industry
\end{tabular}
A B S T R A C T

\begin{abstract}
Management information system (MIS) plays an important role on sharing necessary information within organization. In this paper, we study to find out important factors influencing the implementation of MIS in banking industry. The study designs a questionnaire in Likert scale and distributes it among 253 randomly selected people. Cronbach alpha has been calculated as 0.82 , which is within an acceptable limit. The study uses factor analysis to find important factors and detects six important factors including fear of technology, organizational instability, informal groups, cultural factors, organizational development and understanding that change is always good.
\end{abstract}

(C) 2013 Growing Science Ltd. All rights reserved.

\section{Introduction}

During the past few years, there have been growing interests in implementation of management information system (MIS) in various organizations. However, any MIS implementation may face various challenges such as lack of cooperation from human resource management, complexity in system integration, etc. (Mahmood et al., 1995; Lederer \& Hannu, 1996; Moshref Javadi \& Delshad Dastjerdia, 2011). There are different studies to detect important factors as barriers for MIS implementation. Véronneau and Cimon (2007) examined how to keep robust and effective decision capabilities for firms involved in critical operations using an integrated MIS. They proposed an integrative and cumulative view that was articulated threefold. First, they presented an operations resource management and suggested an integrative approach for decision capabilities as they rest on the interaction between humans and systems. Finally, they derived a decision model and demonstrated that careful consideration could be given to the interplay among humans, systems, and the environment in which they operate.

Renzl (2008) discussed trust in management and knowledge sharing by looking into the mediating effects of fear and knowledge documentation. Rom and Rohde (2007) performed a comprehensive 
review on management accounting and integrated information systems. Some experts believe that MIS implementation in any kind of business model may create different risk in the system and we need to control the risk to prevent any possible damages. Bojanc and Jerman-Blažič (2008), for instance, presented an economic modelling approach to information security risk management. Nevertheless, any MIS implementation has been accompanied by appropriate action plans (Gottschalk, 1999). Factor analysis has been used in banking industry for detecting important factors. Azad and Hassanabadi (2013), for instance, presented an empirical investigation on factors influencing on brand loyalty. In this paper, we present another empirical investigation to find important factors influencing MIS adaptation in banking industry.

\section{The proposed method}

The proposed model of this paper uses factor analysis to determine important factors influencing MIS adaptation in banking industry. The study designs a questionnaire consists of 28 questions and in Likert scale. The study is performed among all existing employees of one of Iranian banks named Bank Eghtesad Novin in city of Tehran, Iran and the sample size is calculated as follows,

$$
n=\frac{N \times z_{\alpha / 2}^{2} \times p \times q}{\varepsilon^{2} \times(N-1)+z_{\alpha / 2}^{2} \times p \times q},
$$

where $N$ is the population size, $p=1-q$ represents the yes/no categories, $z_{\alpha / 2}$ is CDF of normal distribution and finally $\varepsilon$ is the error term. Since we have $p=0.5, z_{\alpha / 2}=1.96$ and $N=2600$, the number of sample size is calculated as $n=253$. Cronbach alpha has been calculated as 0.82 , which is within an acceptable limit. In addition, Kaiser-Meyer-Olkin Measure of Sampling Adequacy is calculated as 0.742 , which is within an acceptable limit and validates the results. Since factor analysis is sensitive on skewness of factors, we have decided to delete seven questions. Fig. 1 demonstrates Scree plot on questions of the survey. In addition, Table 1 shows details of principles component analysis before and after rotation

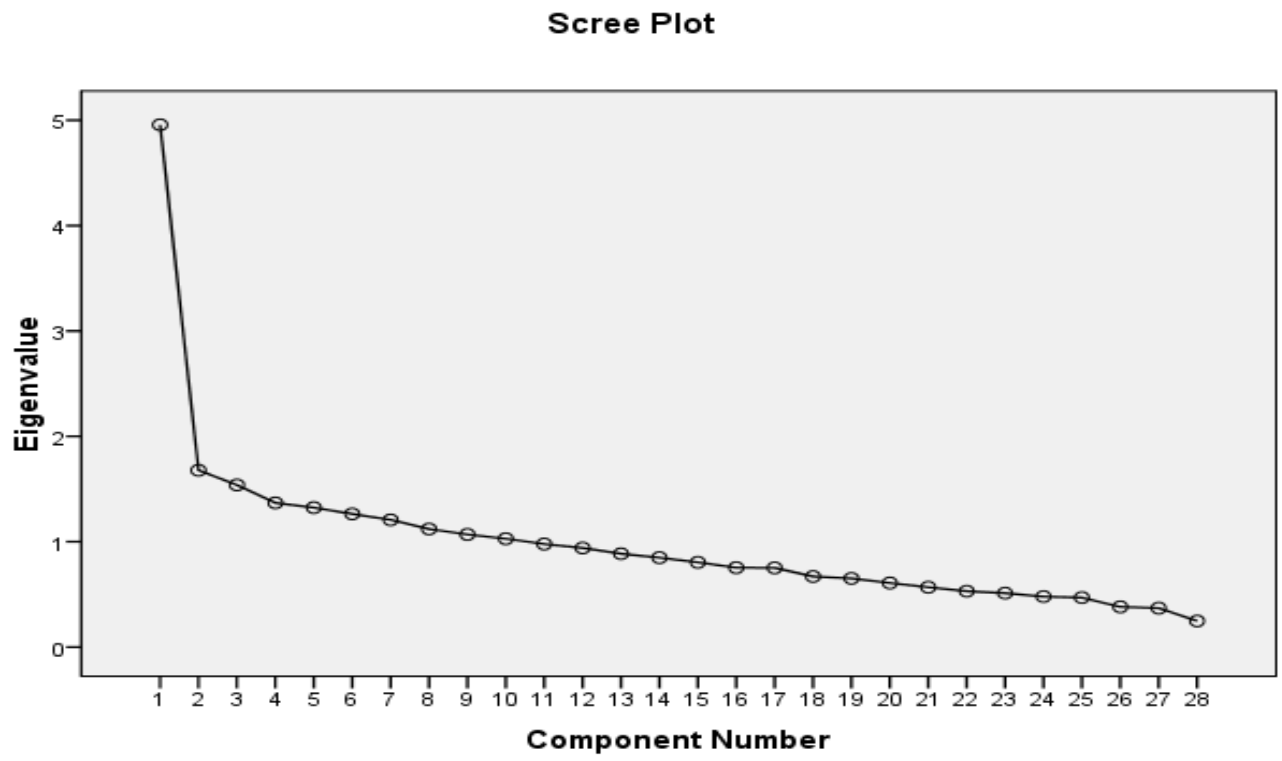

Fig. 1. The Scree plot 
Table 1

The results of principal component analysis with 10 extracted factors

\begin{tabular}{|c|c|c|c|c|c|c|c|c|c|c|c|}
\hline & & 1 & 2 & 3 & 4 & 5 & 6 & 7 & 8 & 9 & 10 \\
\hline Fear of how to use new svstems & VAR00024 & .583 & & & & & & & & & \\
\hline Conflicts with existing norms & VAR00011 & .491 & & & -.344 & & & & & & \\
\hline Fear of losing cooperation with others & VAR00023 & .486 & & & & & & & & & \\
\hline Complexity of new systems & VAR00026 & .482 & & -.396 & & & & & & & \\
\hline Replacing vague system with a determine one & VAR00007 & .477 & & & & & & & & & \\
\hline Lack of cooperation from other people & VAR00028 & .470 & & & & & & & & & \\
\hline Feeling uncomfortable of using new system & VAR00025 & .467 & & -.439 & -.339 & & & & & & \\
\hline Fear of losing iob & VAR00022 & .462 & & & & & & & & & \\
\hline Need for more knowledge & VAR00020 & .460 & & & & & & & & .350 & \\
\hline Fear of losing personal advantage & VAR00015 & .446 & & & .367 & & & & & & -.365 \\
\hline Lack of economic stability & VAR00021 & .440 & & & & & & .336 & & & \\
\hline Feeling that MIS is unnecessary & VAR00002 & .430 & & & & .380 & -.341 & & & & \\
\hline Change in rules and regulations & VAR00017 & .418 & .331 & & & & & .386 & & & \\
\hline Pressure from others to accept MIS & VAR00005 & .414 & & & & -.335 & & & & & .333 \\
\hline Having more risk within organization & VAR00006 & .414 & & & & .344 & & & & & \\
\hline Fear of losing independence & VAR00027 & .409 & & -.400 & & & & & & -.378 & \\
\hline Conflict with group interest & VAR00019 & .447 & .673 & & & & & & & & \\
\hline General culture of having change in organization & VAR00018 & & .586 & & -.389 & & & & & & \\
\hline Having different informal groups & VAR00004 & .393 & & .482 & & & & & & & \\
\hline Fear of losing control of job & VAR00014 & .394 & & & .451 & & & & & & \\
\hline Feeling of wasting too much time to have new MIS system & VAR00001 & & & & & .469 & & & & .428 & \\
\hline Change in job responsibilities & VAR00010 & .377 & & & & .396 & & & & & .333 \\
\hline Fear of expecting difficult task & VAR00012 & & & & & & .524 & & & & \\
\hline Change in habits & VAR00003 & .364 & & & & & & -.433 & & & \\
\hline Change in budgeting system & VAR00009 & .383 & & .367 & & & & -.394 & & & \\
\hline Fear of new training programs & VAR00013 & & & & & & & & .469 & & \\
\hline Fear of losing job security & VAR00008 & .415 & & & & & & & -.419 & & \\
\hline Organizational structure & VAR00016 & .332 & .358 & & & & & & & & .560 \\
\hline
\end{tabular}

Principle component analysis after rotation

\begin{tabular}{|c|c|c|c|c|c|c|c|c|c|c|c|}
\hline & & 1 & 2 & 3 & 4 & 5 & 6 & 7 & 8 & 9 & 10 \\
\hline Feeling uncomfortable of using new svstem & VAR00025 & .621 & & & & & .381 & & & & \\
\hline Fear of how to use new systems & VAR00024 & .608 & & & & & & & & & \\
\hline Complexity of new systems & VAR00026 & .581 & & & & & & & & & \\
\hline Fear of losing independence & VAR00027 & .538 & & & & & & & & & \\
\hline Change in habits & VAR00003 & .532 & & & & & & & & & \\
\hline Fear of losing job & VAR00022 & .509 & & & & & & & & & \\
\hline Having more risk within organization & VAR00006 & & .614 & & & & & & & & \\
\hline Replacing vague system with a determine one & VAR00007 & & .571 & & & & & & & & \\
\hline Change in job responsibilities & VAR00010 & & .530 & & & & & .371 & & & 0.395 \\
\hline Change in budgeting system & VAR00009 & & .529 & .506 & & & & & & & \\
\hline Fear of losing cooperation with others & VAR00023 & & & .601 & & & & & & & \\
\hline Having different informal groups & VAR00004 & & & .593 & & & & & & & \\
\hline Pressure from others to accept MIS & VAR00005 & & & .560 & & & .337 & & & & \\
\hline Fear of losing personal advantage & VAR00015 & & & & .741 & & & & & & \\
\hline Fear of losing control of job & VAR00014 & & & & .704 & & & & & & \\
\hline General culture of having change in organization & VAR00018 & & & & & .879 & & & & & \\
\hline Conflict with group interest & VAR00019 & & & & & .803 & & & & & \\
\hline Lack of economic stability & VAR00021 & & & & & & .758 & & & & \\
\hline Conflicts with existing norms & VAR00011 & & & & & & .571 & & & & \\
\hline Organizational structure & VAR00016 & & & & & & & .735 & & & \\
\hline Change in rules and regulations & VAR00017 & & & & & & .420 & .563 & & & \\
\hline Lack of cooperation from other people & VAR00028 & & & & .370 & & & .382 & & & \\
\hline Feeling of wasting too much time to have new MIS system & VAR00001 & & & & & & & & .685 & & \\
\hline Need for more knowledge & VAR00020 & & & & & & & & 673 & & \\
\hline Feeling that MIS is unnecessary & VAR00002 & & .410 & & & & & & 479 & & \\
\hline Fear of expecting difficult task & VAR00012 & & & & & & & & & 747 & \\
\hline Fear of new training programs & VAR00013 & & & & & & & & & .723 & \\
\hline Fear of losing job security & VAR00008 & & & & & & & & & & 0.721 \\
\hline
\end{tabular}


Based on the results of Table 2 we detect six important factors including fear of technology, organizational instability, informal groups, cultural factors, organizational development and understanding that change is always good.

\section{The results}

In this section, we explain details of our finding on six factors.

\subsection{The first factor: Fear of technology}

The first factor, fear of technology, consists of six sub-factors, which are summarized in Table 2 .

\section{Table 2}

The results of factors associated with fear of technology

\begin{tabular}{|c|c|c|c|c|}
\hline Option & Factor weight & Eigenvalues & $\%$ of variance & Accumulated \\
\hline Change in working habit & 0.63 & & & \\
\hline Fear of losing job & 0.524 & & & \\
\hline Fear of how to use the system & 0.64 & & & \\
\hline Feeling uncomfortable of using the new system & 0.646 & & & \\
\hline Complexity of new systems & 0.69 & 1.326 & 26.512 & 26.512 \\
\hline Fear of losing independence & 0.585 & & & \\
\hline
\end{tabular}

As we can observe from the results of Table 2, complexity of new system is blamed the most as a barrier of MIS implementation in this category followed by feeling uncomfortable and fear of how to use the system.

\subsection{The second factor: Instability in organization}

The second factor, instability in organization, consists of four sub-factors summarized in Table 3 .

\section{Table 3}

The results of factors associated with instability in organization

\begin{tabular}{|c|c|c|c|c|}
\hline Option & Factor weight & Eigenvalues & $\%$ of variance & Accumulated \\
\hline Entering too much risk in organization & 0.709 & & & \\
\hline Replacing uncertainty with certainty & 0.854 & 1.640 & 54.675 & 54.675 \\
\hline Change in responsibilities & 0.684 & & & \\
\hline Change in budging methods & 0.758 & & & \\
\hline
\end{tabular}

According to Table 3, replacing uncertainty with certainty is the most important issue as a barrier of MIS implementation in this category followed by change in budgeting method.

\subsection{The third factor: Informal groups}

The third factor, informal group, consists of three sub-factors, which are summarized in Table 4 as follows,

\section{Table 4}

The results of factors associated with informal groups

\begin{tabular}{|c|c|c|c|c|}
\hline Option & Factor weight & Eigenvalues & $\%$ of variance & Accumulated \\
\hline Existence of various informal groups & 0.719 & & & \\
\hline Pressure from group for accepting changes & 0.729 & & & \\
\hline Fear of losing cooperation with colleagues & 0.827 & 1.857 & 61.916 & 61.916 \\
\hline
\end{tabular}


As we can observe from the results of Table 4, fear of losing cooperation with colleagues is the most important issue as a barrier of MIS implementation in this category followed by pressure from group for accepting changes.

\subsection{The fourth factor: Cultural factors}

The fourth factor, informal group, consists of two sub-factors, which are summarized in Table 5 as follows,

\section{Table 5}

The results of factors associated with cultural factors

\begin{tabular}{lcccc}
\hline Option & Factor weight & Eigenvalues & \% of variance & Accumulated \\
\hline General culture of accepting changes & 0.690 & 1.652 & 55.082 & 55.082 \\
Conflict with existing norms & 0.636 & & & \\
\hline Cronbach alpha $=0.68$ & & & &
\end{tabular}

As we can observe from the results of Table 5, general culture of accepting changes is the most important issue as a barrier of MIS implementation in this category followed by conflict with existing norms.

\subsection{The fifth factor: Organizational change}

The fifth factor, organizational change, consists of three sub-factors, which are summarized in Table 6 as follows,

\section{Table 6}

The results of factors associated with organizational change

\begin{tabular}{lcccc}
\hline Option & Factor weight & Eigenvalues & $\%$ of variance & Accumulated \\
\hline Organizational structure & 0.674 & & & \\
Change in rules and regulations & 0.621 & & & \\
Lack of cooperation & 0.733 & 1.479 & 36.972 & 36.972 \\
\hline
\end{tabular}

Cronbach alpha $=0.64$

As we can observe from the results of Table 6, lack of cooperation is the most important issue as a barrier of MIS implementation in this category followed by organizational structure.

\subsection{The sixth factor: Perception of benefit of having change}

The last factor, perception of benefit of having change, consists of three sub-factors, which are summarized in Table 7 as follows,

\section{Table 7}

The results of factors associated with perception of benefit of having change

\begin{tabular}{lcccc}
\hline Option & Factor weight & Eigenvalues & $\%$ of variance & Accumulated \\
\hline Feeling that the new system is unnecessary & 0.674 & & & \\
Implementation of MIS is a matter of wasting time & 0.621 & & & \\
Feeling needs for learning more & 0.733 & 1.479 & 36.972 & 36.972 \\
\hline
\end{tabular}

Cronbach alpha $=0.61$

As we can observe from the results of Table 7, feeling needs for learning more is the most important issue as a barrier of MIS implementation in this category followed by feeling that the new system is redundant. 


\section{Conclusion}

In this paper, we have presented an empirical investigation to find important factors influencing implementation of management information system in banking systems. The study designed a questionnaire and distributed it among some experts and, using principle component analysis, we have extracted six factors including fear of technology, organizational instability, informal groups, cultural factors, organizational development and understanding that change is always good.

The first factor, fear of technology, consists of six sub-factors where complexity of new system is blamed the most as a barrier of MIS implementation in this category followed by feeling uncomfortable and fear of how to use the system. The second factor, instability in organization, consists of four sub-factors where replacing uncertainty with certainty is the most important issue as a barrier of MIS implementation in this category followed by change in budgeting method. The third factor, informal group, consists of three sub-factors, where fear of losing cooperation with colleagues is the most important issue as a barrier of MIS implementation in this category followed by pressure from group for accepting changes. The fourth factor, informal group, consists of two sub-factors, , general culture of accepting changes is the most important issue as a barrier of MIS implementation in this category followed by conflict with existing norms. The fifth factor, organizational change, consists of three sub-factors, where, lack of cooperation is the most important issue as a barrier of MIS implementation in this category followed by organizational structure. Finally, the last factor, perception of benefit of having change, consists of three sub-factors, where feeling needs for learning more is the most important issue as a barrier of MIS implementation in this category followed by feeling that the new system is redundant.

\section{Acknowledgment}

The authors would like to thank the anonymous referees for their comments on earlier version of this paper.

\section{References}

Azad, N., \& Hassanabadi, M. (2013). An empirical investigation on factors influencing on brand loyalty. Management Science Letters, 3(7), 2113-2118.

Bojanc, R., \& Jerman-Blažič, B. (2008). An economic modelling approach to information security risk management. International Journal of Information Management, 28(5), 413-422.

Gottschalk, P. (1999). Implementation predictors of strategic information systems plans. Information \& Management, 36(2), 77-91.

Lederer, A. L., \& Hannu, S. (1996). Toward a theory of strategic information systems planning. The Journal of Strategic Information Systems, 5(3), 237-253.

Mahmood, M. A., Gowan, M. A., \& Wang, S. P. (1995). Developing a prototype job evaluation expert system: A compensation management application. Information \& management, 29(1), 9-28.

Moshref Javadi, M. H., \& Delshad Dastjerdia, M. (2011). Evaluation effect of management information system implementation on personnel resistance causes in Isfahan power plant management corporation in Iran. Procedia Computer Science, 3, 1296-1303.

Renzl, B. (2008). Trust in management and knowledge sharing: the mediating effects of fear and knowledge documentation. Omega, 36(2), 206-220.

Rom, A., \& Rohde, C. (2007). Management accounting and integrated information systems: A literature review. International Journal of Accounting Information Systems, 8(1), 40-68.

Véronneau, S., \& Cimon, Y. (2007). Maintaining robust decision capabilities: An integrative humansystems approach. Decision support systems, 43(1), 127-140. 